Review Article

\title{
The Heteronuclear Multiple-Quantum Correlation Experiment: Perspective from Classical Vectors, Nonclassical Vectors, and Product Operators
}

\author{
Karen de la Vega-Hernández ${ }^{1}$ and Manuel Antuch ${ }^{2}$ \\ ${ }^{1}$ Departamento de Farmacia, Instituto de Farmacia y Alimentos, Universidad de La Habana, Avenida 23, No. 21425 e/214 and 222, \\ La Coronela, La Lisa, 13600 La Habana, Cuba \\ ${ }^{2}$ Departamento de Química Física, Facultad de Química, Universidad de La Habana, Avenida Zapata y G, Vedado, \\ 10400 La Habana, Cuba
}

Correspondence should be addressed to Karen de la Vega-Hernández; karen16@ifal.uh.cu and Manuel Antuch; manuel.antuch-cubillas@u-psud.fr

Received 16 October 2015; Accepted 6 December 2015

Academic Editor: Guillermo Moyna

Copyright (c) 2016 K. de la Vega-Hernández and M. Antuch. This is an open access article distributed under the Creative Commons Attribution License, which permits unrestricted use, distribution, and reproduction in any medium, provided the original work is properly cited.

\begin{abstract}
It is usually accepted that most 2D-NMR experiments cannot be approached using classical models. Instructors argue that Product Operators (PO) or density matrix formalisms are the only alternative to get insights into complex spin evolution for experiments involving Multiple-Quantum Coherence, such as the Heteronuclear Multiple-Quantum Correlation (HMQC) technique. Nevertheless, in recent years, several contributions have been published to provide vectorial descriptions for the HMQC taking PO formalism as the starting point. In this work we provide a graphical representation of the HMQC experiment, taking the basic elements of Bloch's vector model as building blocks. This description bears an intuitive and comfortable understanding of spin evolution during the pulse sequence, for those who are novice in 2D-NMR. Finally, this classical vectorial depiction is tested against the PO formalism and nonclassical vectors, conveying the didactic advantage of shedding light on a single phenomenon from different perspectives. This comparative approach could be useful to introduce PO and nonclassical vectors for advanced upper-division undergraduate and graduate education.
\end{abstract}

\section{Introduction}

Nuclear magnetic resonance (NMR) has become an indispensable technique in diverse fields such as chemistry [1-4], biochemistry [5-8], structural biology [9-12], materials science [13-15], and biomedicine [16-18]. However, the richness and complexity of NMR, joined to a vast literature, appear intimidating to novice users.

Concerning the educational literature, NMR is a recurrent topic. Many didactic papers are devoted to structure elucidation [19-22], to review media [23, 24], and other applications $[7,8,25,26]$. However, the knowledge behind spin evolution in modern $2 \mathrm{D}-\mathrm{NMR}$ is beyond the grasp of many users since it is not mandatory to interpret NMR spectra.

Most books dealing with 2D-NMR build on Product Operators (PO) or density matrix formalisms to explain spin evolution. Conversely, some other books present simplified treatments of some $2 \mathrm{D}$-NMR experiments through vector representations. Unfortunately, there is a gap between the more elementary books, usually ignoring the most complex theoretical bases, and sophisticated books, treating rigorous methods as almost self-evident [27]. Consequently, an approach lying midway between simple and more elaborated explanations would be useful for didactic purposes.

For such an approach we selected the classical vector (CV) model [28] which permits visualizing some NMR experiments with special comfort. In nowadays (under)graduate teaching, it is widely accepted that most complex 2D-NMR experiments cannot be approached using the classical vector model, in particular those experiments involving MultipleQuantum Coherence (MQC) such as the Heteronuclear Multiple-Quantum Correlation (HMQC). In those cases, 

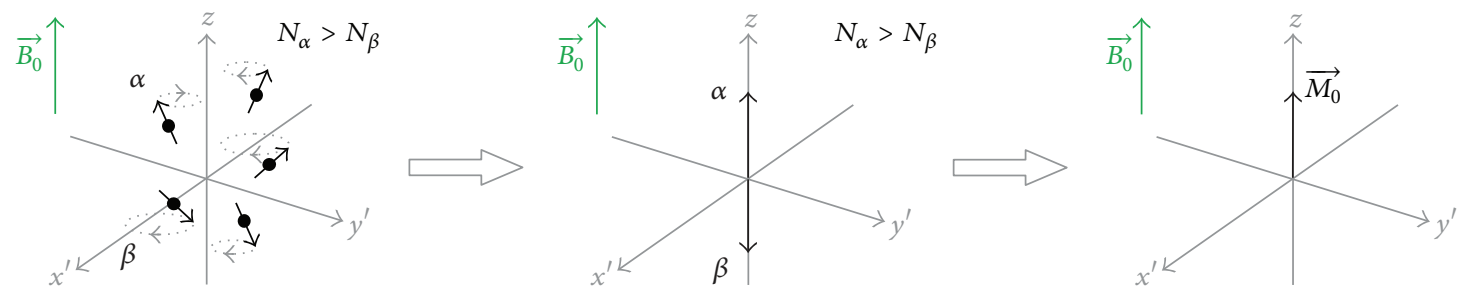

FIGURE 1: Spin-up versus spin-down population difference as a manner to explain the resultant net macroscopic magnetization $\left(\overrightarrow{M_{0}}\right)$ at thermal equilibrium. $N_{\alpha, \beta}$ stand for population of nuclei in the $\alpha$ and $\beta$ spin states, respectively.

instructors argue that PO or density matrix formalisms are the only alternative to get insights into complex spin evolution.

The use of PO for practical purposes is not so complicated since it consists in learning some established rules. However, the physical meaning of mathematical manipulations remains unclear in some cases [29]. This fact prompted some authors to develop graphical representations for PO $[30,31]$. An interesting work describes what was named the nonclassical vector (NCV) model [32]. NCV take PO as the starting point, and its representations are images for each PO. Therefore, in order to comprehend such model, previous knowledge of PO is required, which is beyond the scope of undergraduate and some graduate courses. The great value of NCV resides in offering graphical representations for the equations in PO formalism. Consequently, NCV should be used to accompany $\mathrm{PO}$, and not as an independent model in order to explain multiple-pulse NMR.

The main educational disadvantages of current visual representations of spin states for 2D-NMR experiments are the circumvention of graphical representations for MQC [33] or the use of $\mathrm{PO}$ and wave functions as the starting point $[29,32]$.

In a previous work [28], we provided a classical vector model for the sequence of events occurring during the Heteronuclear Single-Quantum Correlation (HSQC) experiment and the further comparison with PO. In this paper, we present a graphical representation of the HMQC experiment, following the same spirit as in our previous publication. In addition, we extend the comparative analysis to NCV and provide a more exhaustive and rigorous view of MQC evolution during the evolution period.

The classical representation of the HMQC allows an intuitive understanding as far as possible of the resulting spectrum appearance without the use of quantum mechanics. The further correspondence with PO and NCV allows for the comparison of the same phenomenon from the perspective of different models. Such comparison permits validating the proposed classical vector model as a pedagogical tool for introducing 2D-NMR [28].

\section{Some Initial Comments}

The specialized literature offers many ways of presenting NMR. It is well recognized that NMR is a quantum phenomenon. However, classical mechanic approaches are often preferred because of its simpler nature and inherent intuitiveness [34]. Such methodology is consistent and in some cases presents excellent correspondence with more rigorous treatments [28].

Henceforward, only nuclei with $I=1 / 2$ shall be considered. It is well established that, for a single nucleus having $I=1 / 2$, a measurement of $\mu$ gives only one of two possible orientations, namely, $\alpha$ and $\beta$, for the projection of $\mu$ along the field direction.

However, this is not the case in systems composed by many spins, in which the average orientation associated with $\mu$ in a magnetic field (i.e., the expectation value in quantum mechanics) gives bulk macroscopic magnetization that does not have quantized values $[34,35]$. Deepening into the orientations of individual spins in the magnetic field is unnecessary in this context. Accurately speaking, the system is in a mixed state and there are innumerable microscopic configurations that would result in the same mixed state. Suffice it to say that, at equilibrium, the polarization of a population of spin-up versus spin-down nuclei is one possibility that gives the correct density matrix for the particular mixed state (Figure 1).

When a strong magnetic field is established $\left(\overrightarrow{B_{0}}\right)$, individual magnets precess about the field at the Larmor frequency (Figure 1, left). The origin of NMR lies on the torque that a linearly polarized electromagnetic pulse $\left(\overrightarrow{B_{1}}\right)$ with radiofrequency wavelength exerts on the macroscopic magnetization $\left(\overrightarrow{M_{0}}\right)$. Such pulse makes $\overrightarrow{M_{0}}$ rotate from $z$-axis towards the $x^{\prime} y^{\prime}$ plane (also known as transverse plane), giving rise to the observable transverse magnetization. In our classical vector description of the HMQC explained below, we propose to understand the rotation of $\overrightarrow{M_{0}}$ as the simultaneous rotation of the $\alpha$ and $\beta$ components [35].

\section{General Description of the HMQC Pulse Sequence}

The pulse sequence of the HMQC experiment may be appreciated on top of Figure 2. Like all 2D-NMR experiments, the HMQC possesses four stages known as preparation, evolution, mixing, and detection. The preparation stage consists in $90_{x}^{\circ}$ pulse to both nuclei, separated by a delay time $\Delta$. Then comes the evolution period, which consists in a $180_{x}^{\circ}$ pulse to proton in the middle. The $90_{x}^{\circ}$ pulse applied to carbon 

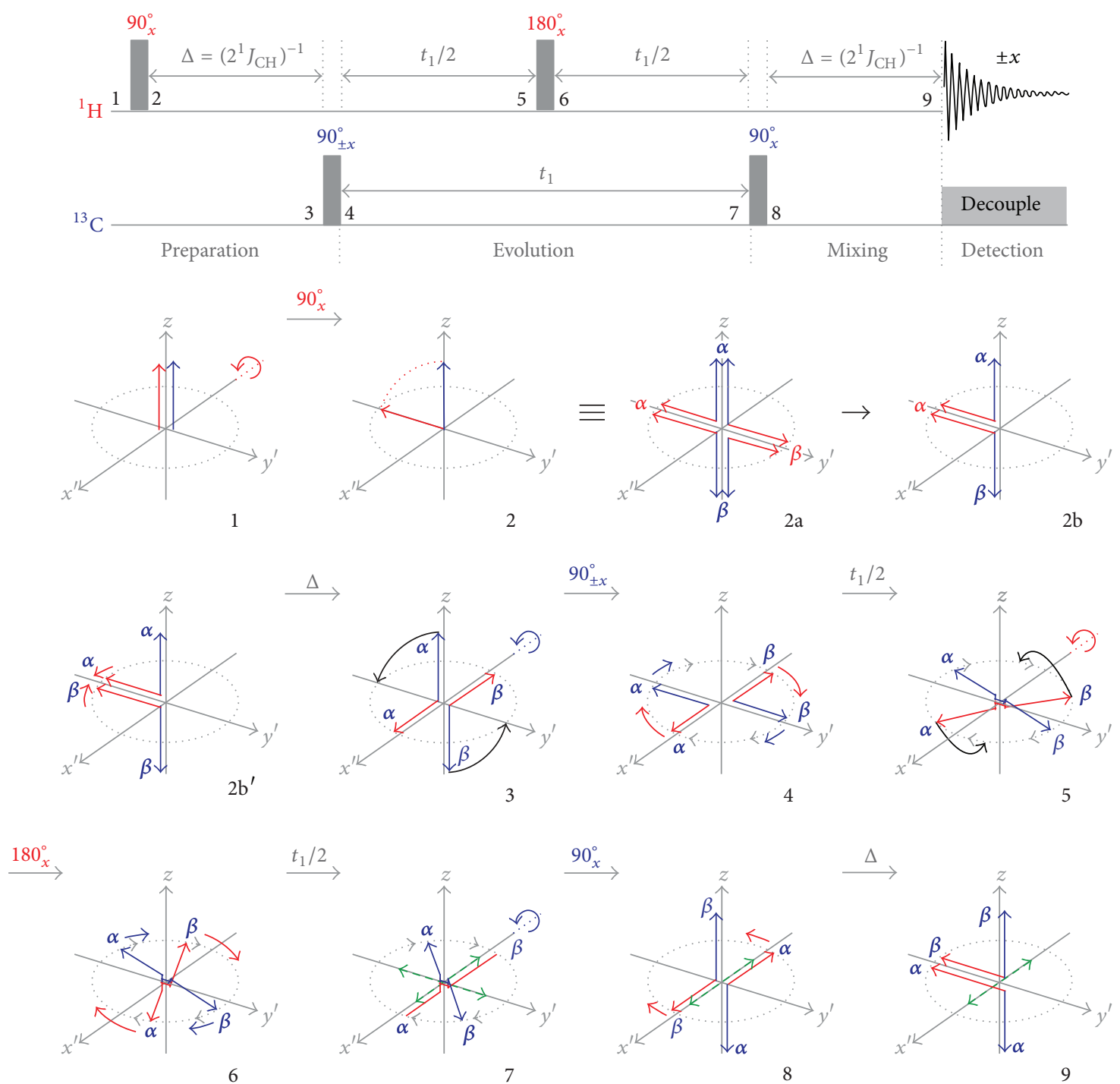

FIgURE 2: The pulse sequence of HMQC is presented on top. Pulses are designated by the rotation angle, in degrees, with a subscript indicating the axis. Magnetization evolution is presented in schemes below. Thick arrows represent net magnetization and thin arrows represent vector components. Red color is used for proton, while blue is used for carbon. Green dashed arrows in scheme 7 represent the projection of carbon components into the $x$ - and $y$-axes. Further insights concerning the interpretation of schemes 7-9 shall be given in the section devoted to a comparative analysis.

and another delay time $\Delta$ constitute the mixing period, before the detection at the end [36].

Henceforward, the terms proton and carbon shall refer to ${ }^{1} \mathrm{H}$ and ${ }^{13} \mathrm{C}$. In the vector schemes of Figure 2 and throughout this paper, red color corresponds to proton, while blue corresponds to carbon. Thick arrows represent net magnetization and thin arrows stand for vector components. All subsequent vector diagrams are drawn using a right-handed rotating coordinate system. In this work, we accommodated the signs of the CV model in order to agree with PO [28], rather than in the opposite sense, as it has been presented in other publications [29]. Therefore, after the initial $90_{x}^{\circ}$ pulse to proton, net magnetization flips towards $-y^{\prime}$. Pulses to proton and carbon are assumed to be on resonance to avoid scheme complications due to offset evolution.

\section{Classical Vector Description}

4.1. Preparation. The first $90_{x}^{\circ}$ pulse to proton creates proton transverse magnetization along $-y^{\prime}$-axis, while carbon net magnetization remains along $+z$-axis (longitudinal magnetization) (Figure 2, scheme 2).

In order to provide a coherent vectorial explanation for the experiment, the spin state of Figure 2 scheme 2 shall be represented by its vector components as in Figure 2 scheme 2a. Figure 2 scheme 2 a may be interpreted in the following manner: there are two components of carbon magnetization, namely, $\alpha_{\mathrm{C}}$ and $\beta_{\mathrm{C}}$ (represented in blue), (approximately) half of $\alpha_{\mathrm{C}}$ is coupled to protons with spin $\alpha$ (being the spin state $\left.\alpha_{\mathrm{C}} \alpha_{\mathrm{H}}\right)$, and the other half is coupled to protons with spin $\beta$ (state $\alpha_{\mathrm{C}} \beta_{\mathrm{H}}$ ). Similar reasoning may be performed for carbon 
TABLE 1: Representation and significance of one-spin and two-spin operators [28, 37].

\begin{tabular}{|c|c|}
\hline Product Operator & Coherence name \\
\hline $\mathrm{I}_{\mathrm{z}}, \mathrm{S}_{\mathrm{z}}$ & Longitudinal $I$-spin $(S$-spin) magnetization \\
\hline$I_{x}, I_{y}, S_{x}, S_{y}$ & Transverse $I$-spin ( $S$-spin) magnetization \\
\hline $2 \mathrm{I}_{\mathrm{z}} \mathrm{S}_{\mathrm{z}}$ & Nonequilibrium longitudinal two-spin magnetization \\
\hline $2 I_{x} S_{z}, 2 I_{y} S_{z}$ & Transverse $I$-spin magnetization antiphase with respect to the coupling to $S$-spin \\
\hline $2 I_{z} S_{x}, 2 I_{z} S_{y}$ & Transverse $S$-spin magnetization antiphase with respect to the coupling to $I$-spin \\
\hline $2 I_{x} S_{y}, 2 I_{y} S_{x}, 2 I_{x} S_{x}, 2 I_{y} S_{y}$ & Multiple-Quantum Coherence \\
\hline
\end{tabular}

with spin $\beta$. Further schemes simplifications are adopted here to avoid figure crowding. Figure 2 scheme 2 a transforms into Figure 2 scheme $2 b$, by not representing carbons coupled to $\beta$-spin protons. Naturally, any subsequent analysis of spin evolution concerns the unrepresented $\alpha_{\mathrm{C}} \beta_{\mathrm{H}}$ and $\beta_{\mathrm{C}} \beta_{\mathrm{H}}$ in the same way.

Just after the $90_{x}^{\circ}$ pulse, proton components lying along $-y^{\prime}$, split according to the coupling with the carbon partner. Protons coupled to carbons with spin $\beta$ shall move towards $-x^{\prime}$, while those coupled to carbons with spin $\alpha$ shall move towards $+x^{\prime}$ (Figure 2, scheme $2 b^{\prime}$ ). The system is allowed to evolve a delay time $\Delta$ equal to $\left(2^{1} J_{\mathrm{CH}}\right)^{-1}$, where ${ }^{1} J_{\mathrm{CH}}$ stands for the one-bond coupling constant between a carbon and the proton directly attached to it. After this time, proton components are in opposite directions. In this state, vectors are said to be antiphase to one another (Figure 2, scheme 3 ). The subsequent $90_{x}^{\circ}$ pulse to carbon rotates the carbon components from $\pm z$ - to $\pm y^{\prime}$-axis (Figure 2, scheme 4). The state resulting from this pulse is known as MQC, which is unobservable, and may be thought as simultaneous transverse magnetization of both nuclei. Figure 2 scheme 4 is perhaps the most inaccurate part of this vectorial explanation. The fact is that MQC is undetectable, and the vector model cannot account for such feature.

4.2. Evolution. At this point, proton and carbon components are antiphase in the $x^{\prime}$ - and $y^{\prime}$-axes, respectively (Figure 2, scheme 4). During MQC, the heteronuclear coupling does not evolve [37], which is another feature that cannot be accounted by the classical vector model. Hence, both proton and carbon offsets, along with homonuclear coupling (not shown in Figure 2), are the observables evolved during $t_{1}$ (Figure 2, schemes 4 to 7 ). The $180_{x}^{\circ}$ pulse to proton in the middle of the evolution period serves to refocus its offset. Thus, carbon offsets, modulated by proton homonuclear coupling, shall appear in the indirect frequency $F_{1}$.

As a matter of fact, speaking about carbon or proton offsets independently is not so rigorous. Further explanations shall be given at the end of this paper.

4.3. Mixing. The following $90_{x}^{\circ}$ pulse brings carbon components to $\pm z$-axis whilst proton vectors are still forming an angle of $\pi$ radians in-between (Figure 2, scheme 8). As a result, MQC is turned into Single-Quantum Coherence (SQC), which means that now only one type of nucleus has transverse coherence. After another delay time $\Delta$ equal to $\left(2^{1} J_{\mathrm{CH}}\right)^{-1}$, proton vectors evolve under heteronuclear coupling until both components are in-phase (Figure 2, scheme 9). It is worth noting that carbon components in Figure 2 scheme 8 are inverted with respect to those represented in Figure 2 scheme $2 b^{\prime}$; that is, an inversion of carbon population has occurred. This implies that the proton having initially a $\beta$-carbon as coupling partner has now an $\alpha$ carbon as partner and vice versa. Consequently, both proton components finally align along $-y^{\prime}$.

This vectorial depiction cannot explain the origin of the characteristic cross-peak between carbons and protons directly attached; it constitutes an inherent limitation of vectorial representations, clarified by the PO formalism.

The detection period starts then. Proton offset and homonuclear coupling evolve and are observed in $F_{2}$.

\section{Some Other Perspectives of the HMQC}

5.1. Product Operators. Product Operators (PO) constitute the representation of the spin density matrix $(\rho)$ into a comfortable basis. Therefore, any spin state may be expressed as a combination of these basis operators. Table 1 presents PO for one-spin and two-spin systems and a description of their significance. From here on, PO shall be written in bold and proton and carbon operators are represented by $\mathbf{I}$ and $\mathbf{S}$, respectively.

The operator $\mathbf{I}_{\mathbf{z}}\left(\right.$ or $\mathbf{S}_{\mathbf{z}}$ ) means that the nucleus whose operator is denoted by $I$ (or $S$ ) presents (longitudinal) magnetization along the $z$-axis. The operator $2 I_{y} S_{z}$ represents transverse magnetization of the nucleus $I$ along $y$, which is antiphase with respect to the coupling to $S$. In the same way, the operator $2 \mathbf{I}_{\mathbf{z}} \mathbf{S}_{\mathbf{y}}$ stands for antiphase magnetization of the nucleus $S$ along $y$, with respect to the coupling to $I$. The factor of 2 in these operators is a normalization factor. Multiplequantum operators, like $2 \mathbf{I}_{\mathbf{x}} \mathbf{S}_{\mathbf{y}}$, are comprised of transverse operators for both nuclei at the same time. However, these do not represent transverse magnetization. In fact, there is no transverse magnetization in spin states represented by multiple-quantum operators. A physical description for the operator $2 \mathbf{I}_{\mathbf{z}} \mathbf{S}_{\mathbf{z}}$ is not straightforward; it has been defined as a particular type of nonequilibrium population distribution $[28,37]$.

When the relevant Hamiltonian does not depend on time, the equation of motion for the density matrix is given by [37, 38]

$$
\boldsymbol{\rho}(t)=e^{-i \mathbf{H} t} \boldsymbol{\rho}(0) e^{i \mathbf{H} t}
$$


Equation (1) possesses a limited number of solutions [37], which are commonly written succinctly with a shorthand notation. It consists in an arrow connecting the old and new PO; over the arrow the relevant Hamiltonian is written. For example, (2) represents a pulse to the nucleus $I$ about $x^{\prime}$, which causes a rotation by an angle $\omega t_{p}$ (the Hamiltonian being $\left.\omega t_{p} \mathbf{I}_{\mathbf{x}}\right)$. If $\omega t_{p}=90^{\circ}$, then $\cos \omega t_{p}=0$ and $\sin \omega t_{p}=1$. The net result is precession of $+z$ magnetization towards $-y^{\prime}$ with a sinusoidal dependence on the flip angle $\omega t_{p}$. Besides, if a pulse is applied to an operator ( (say about $y^{\prime}$ ), the operator remains unaltered [28]:

$$
\mathbf{I}_{\mathbf{z}} \stackrel{\omega t_{p} \mathbf{I}_{\mathbf{x}}}{\longrightarrow} \mathbf{I}_{\mathbf{z}} \cos \omega t_{p}-\mathbf{I}_{\mathbf{y}} \sin \omega t_{p} .
$$

The full sequence of events for the HMQC described in terms of PO appears in the second column of Table 2. For an easier comparison with $\mathrm{CV}$, the first column of Table 2 corresponds to the numbered schemes in Figure 2.

5.2. Nonclassical Vectors. Nonclassical vectors (NCV) were developed with the purpose of making PO accessible and visual to a wide audience [32]. The model assigns a nonclassical vector to each PO and was established to comply with the physical meaning of $\mathrm{PO}$, thus providing valuable physical representations of spin states. In this paper, we shall not discuss all existing $\mathrm{NCV}$, since the purpose is to analyze the HMQC in terms of CV, NCV, and PO. The relevant NCV for this work appear in the third column of Table 2.

NCV representation of one-spin operators is two parallel single-headed arrows along the corresponding axis. Conversely, two-spin operators are represented with doubleheaded arrows. The reasons behind the selection of such representation are well explained and acceptable with ease $[32,39]$. For example, the NCV correspondent to the PO $\mathbf{I}_{\mathbf{z}}$ (in a system of two coupled nuclei) possesses two singleheaded arrows in order to account for the two possible spin projections of the coupling partner. Conversely, all two-spin operators arise from pulses to the NCV representation of $\mathbf{I}_{\mathbf{z}} \mathbf{S}_{\mathbf{z}}$. For further details, we suggest seeing the original paper [32].

NCV can accommodate offset evolution in a way similar to that of CV. However, in the case of scalar coupling evolution, NCV are often unintuitive because of its subordination to PO. For instance, in Table 2 (third column), concerning the transformation of scheme 2 into 3 (i.e., the splitting of $-\mathbf{I}_{\mathbf{y}}$ because of heteronuclear coupling), the reader might ask why the blue arrows appear in scheme 3 since these were not represented in scheme 2. Nevertheless, $\mathrm{NCV}$ are very valuable since they provide us with consistent representations, even though inherent limitations arise from any vectorial representation of such phenomena.

\section{Comparative Analysis}

Table 2 presents the full sequence of events of the HMQC experiment. The numbering in the first column corresponds to the schemes in Figure 2. The second and third columns correspond to the equivalent PO terms and NCV diagrams, respectively.
6.1. Preparation. The first $90_{x}^{\circ}$ pulse converts $\mathbf{I}_{\mathbf{z}}$ into $-\mathbf{I}_{\mathbf{y}}$ in agreement with Figure 2 schemes 1 and 2 and (2). These schemes are quite similar to the related NCV diagram. The main difference is that the corresponding NCV presents two arrows to represent each spin (only spin $I$ in this case) instead of one arrow for each spin as in Figure 2 schemes 1 and 2.

Then, PO $-\mathbf{I}_{\mathbf{y}}$ evolves under heteronuclear coupling during a delay time that permits complete conversion of inphase into antiphase magnetization represented by the PO $2 \mathbf{I}_{\mathbf{x}} \mathbf{S}_{\mathbf{z}}$, as presented in Figure 2 scheme 3; NCV represent this state in a similar manner. The other $90_{x}^{\circ}$ pulse to spin $S$ turns $\mathbf{2} \mathbf{I}_{\mathbf{x}} \mathbf{S}_{\mathbf{z}}$ into $-\mathbf{2} \mathbf{I}_{\mathbf{x}} \mathbf{S}_{\mathbf{y}}$, in accordance with Figure 2 scheme 4 , and the related nonclassical vector.

6.2. Evolution. During this period, heteronuclear coupling does not evolve and the offset of proton is refocused due to the spin echo sequence. Therefore, only the offset of carbon modulated by homonuclear coupling evolves. The PO term correspondent to Figure 2 schemes 5 to 7 represents evolution of the corresponding PO $\mathbf{- 2} \mathbf{I}_{\mathbf{x}} \mathbf{S}_{\mathbf{y}}$ under the offset of $S\left(\Omega_{S}\right)$ after an evolution time equal to $t_{1}$. As a result, the $\alpha$ and $\beta$ components of proton are antiphase along the $x^{\prime}$-axis. In addition, the $\alpha$ and $\beta$ components of carbon are antiphase as well, represented as a combination of $2 \mathbf{I}_{\mathbf{x}} \mathbf{S}_{\mathbf{x}}$ and $2 I_{x} S_{y}$. In corresponding Figure 2 scheme 7, this state may be observed, which is represented by NCV as a combination of two diagrams.

6.3. Mixing. At this point, the $\alpha$ and $\beta$ components of both nuclei are antiphase in the $x^{\prime} y^{\prime}$ plane. The next $90_{x}^{\circ}$ pulse to carbon converts MQC into SQC $\left(2 \mathbf{I}_{\mathbf{x}} \mathbf{S}_{\mathbf{z}}\right)$, as may be observed in Figure 2 scheme 8. However, PO uncovers further unobservable terms $\left(\mathbf{2} \mathbf{I}_{\mathbf{x}} \mathbf{S}_{\mathbf{x}}\right.$ in this case) presented as green dashed arrows in Figure 2 scheme 8. Such MQC term appears in the PO formalism and in NCV. Its apparition in classical vector diagrams is not so obvious and follows the same reasoning presented by us (Figure 2, schemes 7-9) [28].

Namely, carbon magnetization may be divided into its respective components along $x^{\prime}$ - and $y^{\prime}$-axes (Figure 2, scheme 7). Considering that the $90_{x}^{\circ}$ pulse does not affect the component along $x^{\prime}$, such component survives in the transverse plane, while the component onto $y^{\prime}$ flips towards $+z$ (Figure 2 scheme 8 ). Such classical picture presents all these features in a single scheme (Figure 2 scheme 7 ) and is perhaps more adequate than the NCV representation where it should be observed as the superposition of two different schemes (Table 1, scheme 8).

The term $\left(2 \mathbf{I}_{\mathbf{x}} \mathbf{S}_{\mathbf{z}} \cos \Omega_{S} t_{1}\right)$ then evolves to in-phase magnetization and finally results in $\left(-\mathbf{I}_{\mathbf{y}} \cos \Omega_{S} t_{1}\right)$, which is the final observable, as presented in Figure 2 scheme 9 and the corresponding NCV. The final result is proton transverse magnetization along $-y^{\prime}\left(-\mathbf{I}_{\mathbf{y}}\right)$ evolving at the offset of carbon $\left(\Omega_{S}\right)$, giving rise to the characteristic cross-peak between carbons and the directly attached protons. The spectrum contains an in-phase doublet in $F_{2}$ centered at the offset of proton and in $F_{1}$ at the offset of carbon, broadened by proton homonuclear coupling. 
TABLE 2: Relation of vector schemes, PO, and NCV diagrams for the HMQC experiment. Red color is employed for proton and blue for carbon.

\begin{tabular}{|c|c|c|}
\hline Sch. & Product Operator terms [37] & Nonclassical vector diagrams $[31,32]$ \\
\hline 1 & $I_{z}$ & \\
\hline 2 & $\stackrel{\left(90^{\circ}\right) \mathbf{I}_{\mathbf{x}}}{\longrightarrow}-\mathbf{I}_{\mathbf{y}}$ & \\
\hline 3 & $\stackrel{2 \pi I_{I S} \Delta \mathbf{I}_{\mathbf{z}} \mathbf{S}_{\mathbf{z}} ; \Delta=1 /\left(2^{1} I_{I S}\right)}{\longrightarrow} 2 \mathbf{I}_{\mathbf{x}} \mathbf{S}_{\mathbf{z}}$ & \\
\hline 4 & $\stackrel{\left(90^{\circ}\right) \mathbf{S}_{\mathrm{x}}}{\longrightarrow}-2 \mathbf{I}_{\mathbf{x}} \mathbf{S}_{\mathbf{y}}$ & \\
\hline
\end{tabular}

5-7

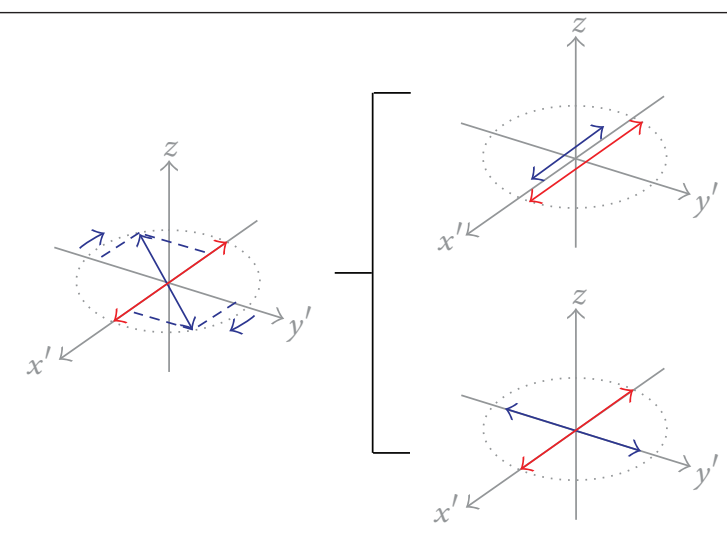

8

$\stackrel{\left(90^{\circ}\right) \mathbf{S}_{\mathbf{x}}}{\longrightarrow}-2 \mathbf{I}_{\mathbf{x}} \mathbf{S}_{\mathbf{z}} \cos \Omega_{S} t_{1}+2 \mathbf{I}_{\mathbf{x}} \mathbf{S}_{\mathbf{x}} \sin \Omega_{S} t_{1}$

9

$\stackrel{2 \pi I_{I S} \Delta \mathbf{I}_{\mathbf{z}} \mathbf{S}_{\mathbf{z}} ; \Delta=1 /\left(2^{1} J_{I S}\right)}{\longrightarrow}-\mathbf{I}_{\mathbf{y}} \cos \Omega_{S} t_{1}+2 \mathbf{I}_{\mathbf{x}} \mathbf{S}_{\mathbf{x}} \sin \Omega_{S} t_{1}$

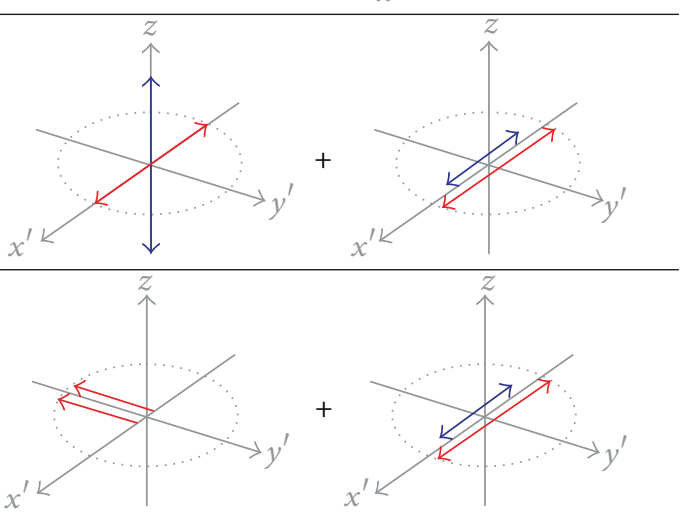




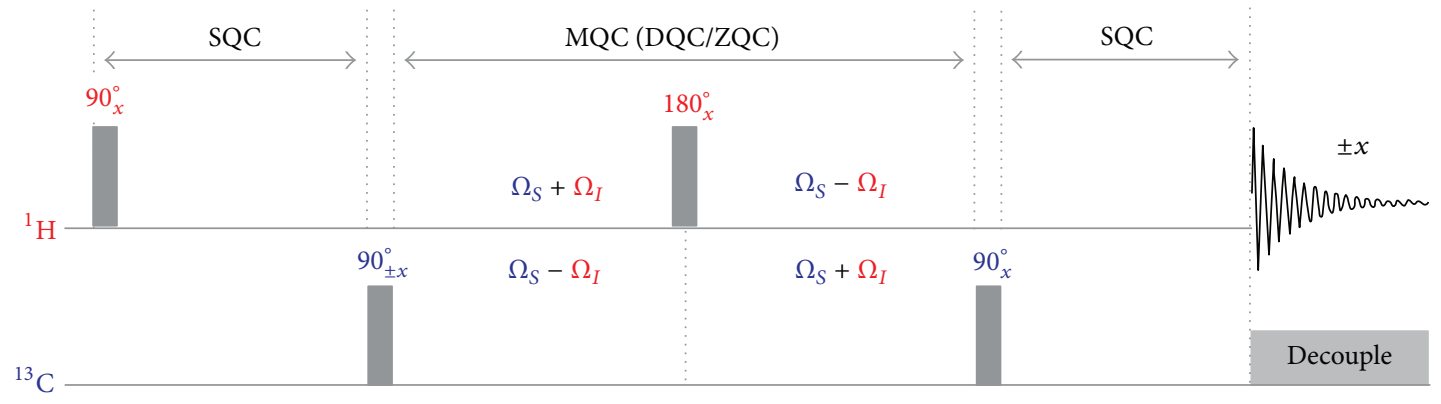

FIgURE 3: Simple representation of offset evolution of Multiple-Quantum Coherence (MQC) during the evolution period. Observe how Double-Quantum Coherence (DQC) evolves at $\left(\Omega_{S}+\Omega_{I}\right)$ while Zero-Quantum Coherence (ZQC) evolves at $\left(\Omega_{S}-\Omega_{I}\right)$. The $180_{x}^{\circ}$ pulse to protons converts DQC into ZQC and vice versa, thus leading to cancellation of $\Omega_{I}$; therefore, the final output of the evolution period is $\Omega_{S}$ [40].

6.4. A Closer Look to the Evolution Period. The evolution period is not simple as treated above. MQC should be more properly represented as a combination of Zero-Quantum Coherence (ZQC) and Double-Quantum Coherence (DQC). ZQC and DQC are combinations of two-spin operators as represented by [37]

$$
\begin{aligned}
& \mathrm{DQ}_{\mathrm{x}}=0.5\left(2 \mathrm{I}_{\mathrm{x}} \mathrm{S}_{\mathrm{x}}-2 \mathrm{I}_{\mathrm{y}} \mathrm{S}_{\mathrm{y}}\right) \text {, } \\
& D Q_{y}=0.5\left(2 \mathrm{I}_{\mathbf{x}} \mathrm{S}_{\mathbf{y}}+2 \mathrm{I}_{\mathrm{y}} \mathrm{S}_{\mathrm{x}}\right) \text {, } \\
& Z_{\mathbf{x}}=0.5\left(2 \mathrm{I}_{\mathbf{x}} \mathrm{S}_{\mathrm{x}}+2 \mathrm{I}_{\mathbf{y}} \mathrm{S}_{\mathrm{y}}\right) \text {, } \\
& Z_{\mathbf{y}}=0.5\left(2 \mathrm{I}_{\mathbf{y}} \mathrm{S}_{\mathrm{x}}-2 \mathrm{I}_{\mathrm{x}} \mathrm{S}_{\mathrm{y}}\right) \text {. }
\end{aligned}
$$

At the beginning of the evolution period, the relevant $\mathrm{PO}$ is $-2 I_{x} S_{y}$. To express its evolution in terms of MQC operators, observe that $2 I_{\mathbf{x}} S_{\mathbf{y}}$ is present in (4) and (6) only. Thus, (6) minus (4) and rearrangement yields

$$
-2 I_{x} S_{y}=Z Q_{y}-D Q_{y}
$$

which is the representation of the spin state of the system, at the beginning of the evolution period, in terms of multiplequantum operators.

ZQC and DQC operators evolve according to

$$
\begin{aligned}
\mathbf{Z Q}_{\mathbf{y}} \stackrel{\left(\mathbf{S}_{\mathbf{z}} \Omega_{S}+\mathbf{I}_{\mathbf{z}} \Omega_{I}\right) t}{\longrightarrow} & \mathbf{Z Q}_{\mathbf{y}} \cos \left(\Omega_{S}-\Omega_{I}\right) t \\
& -\mathbf{Z Q}_{\mathbf{x}} \sin \left(\Omega_{S}-\Omega_{I}\right) t \\
\mathbf{D Q}_{\mathbf{y}} \stackrel{\left(\mathbf{S}_{\mathbf{z}} \Omega_{S}+\mathbf{I}_{\mathbf{z}} \Omega_{I}\right) t}{\longrightarrow} & \mathbf{D Q}_{\mathbf{y}} \cos \left(\Omega_{S}+\Omega_{I}\right) t \\
& -\mathbf{D Q}_{\mathbf{x}} \sin \left(\Omega_{S}+\Omega_{I}\right) t .
\end{aligned}
$$

As observed above, DQC terms evolve at an effective frequency $\left(\Omega_{S}+\Omega_{I}\right)$ and ZQC terms evolve at $\left(\Omega_{S}-\Omega_{I}\right)$. Therefore, the evolution of MQC, that is, DQC and ZQC, should be understood as the complex and correlated movement of both nuclei, rather than the simplistic view of independent and simultaneous evolution of transverse magnetization of both nuclei.
Figure 3 represents the stages of the HMQC where SQC and MQC take place. In the first half of the evolution period (before the $180_{x}^{\circ}$ pulse), DQC and ZQC operators evolved according to $\left(\Omega_{S}+\Omega_{I}\right)$ and $\left(\Omega_{S}-\Omega_{I}\right)$, respectively (Figure 3 ). The $180_{x}^{\circ}$ pulse to proton in the middle of this period causes the transformation of DQC into ZQC and vice versa (Figure 3, second half of the evolution period). As the overall result, proton offsets cancel and the net output shall be the evolution of carbon offset.

Consequently, in order to avoid entanglement in the description of the evolution period, some authors treat it without mentioning $\mathrm{DQC}$ and $\mathrm{ZQC}$, as in the previous section [37], while some others make full and rigorous descriptions [36]. The thoroughness of the treatment depends on the deepness of the corresponding course.

\section{Conclusion}

This work presented the 2D-NMR HMQC from the perspective of three different models. Such comparative approach is useful to introduce advanced undergraduate and graduate students to the theory behind spin evolution in $2 \mathrm{D}$ NMR. This approach is particularly appropriate for those who have little background in quantum mechanics since it builds on a classical framework. Throughout the work, the limitations of the classical vector model became evident, and more sophisticated formalisms (Product Operators and nonclassical vectors) were helpful to account for such gaps in the explanations. This classical model does not offer all the answers for multiple-pulse experiments. For example, it fails to explain basic concepts such as polarization transfer. Therefore, attempts to extend this model to other experiments should be performed with care. Nevertheless, to provide graphical depictions of spin quantum states (taking the HMQC as example) is a valuable tool if the researcher is aware of the boundaries of the particular model used.

\section{Disclosure}

Manuel Antuch currently works at Equipe de Recherche et d'Innovation en Electrochimie pour l'Energie (ERIEE), Institut de Chimie Moléculaire et des Matériaux d'Orsay 
(ICMMO), Université Paris-Saclay-Paris-Sud, rue du Doyen Georges Poitou, Bâtiment 410, 91400 Orsay, France.

\section{Conflict of Interests}

The authors declare that there is no conflict of interests regarding the publication of this paper.

\section{Acknowledgments}

The authors wish to gratefully thank Osmany Cuesta Rubio and Carlos Pérez Martínez for encouraging the love for NMR spectroscopy. They are also thankful to Pedro Ortiz del Toro for pleasant introductions to quantum mechanics.

\section{References}

[1] B. Procacci, Y. Jiao, M. E. Evans, W. D. Jones, R. N. Perutz, and A. C. Whitwood, "Activation of $\mathrm{B}-\mathrm{H}, \mathrm{Si}-\mathrm{H}$, and $\mathrm{C}-\mathrm{F}$ Bonds with $\mathrm{Tp}^{\prime} \mathrm{Rh}\left(\mathrm{PMe}_{3}\right)$ complexes: kinetics, mechanism, and selectivity," Journal of the American Chemical Society, vol. 137, no. 3, pp. 1258-1272, 2015.

[2] K. S. Feldman, I. Y. Gonzalez, and C. M. Glinkerman, "Intramolecular $[3+2]$ cyclocondensations of alkenes with indolidenes and indolidenium cations," Journal of the American Chemical Society, vol. 136, no. 43, pp. 15138-15141, 2014.

[3] S. E. Ashbrook and S. Sneddon, "New methods and applications in solid-state NMR spectroscopy of quadrupolar nuclei," Journal of the American Chemical Society, vol. 136, no. 44, pp. 1544015456, 2014.

[4] S. S. Zalesskiy, E. Danieli, B. Blümich, and V. P. Ananikov, "Miniaturization of NMR systems: desktop spectrometers, microcoil spectroscopy, and 'nMR on a Chip' for chemistry, biochemistry, and industry," Chemical Reviews, vol. 114, no. 11, pp. 5641-5694, 2014.

[5] R. Schneider, D. Maurin, G. Communie et al., "Visualizing the molecular recognition trajectory of an intrinsically disordered protein using multinuclear relaxation dispersion NMR," Journal of the American Chemical Society, vol. 137, no. 3, pp. 1220-1229, 2015.

[6] M. R. Preimesberger, A. Majumdar, T. Aksel et al., "Direct NMR detection of bifurcated hydrogen bonding in the $\alpha$-helix N-caps of ankyrin repeat proteins," Journal of the American Chemical Society, vol. 137, no. 3, pp. 1008-1011, 2015.

[7] L. H. Lucas and C. K. Larive, "Measuring ligand-protein binding using NMR diffusion experiments," Concepts in Magnetic Resonance Part A, vol. 20, no. 1, pp. 24-41, 2004.

[8] J. C. Lindon, J. K. Nicholson, E. Holmes, and J. R. Everett, "Metabonomics: metabolic processes studied by NMR spectroscopy of biofluids," Concepts in Magnetic Resonance, vol. 12, no. 5, pp. 289-320, 2000.

[9] T. Jacso, B. Bardiaux, J. Broecker et al., "The mechanism of denaturation and the unfolded state of the $\alpha$-helical membraneassociated protein Mistic," Journal of the American Chemical Society, vol. 135, no. 50, pp. 18884-18891, 2013.

[10] K. Berlin, C. A. Castañeda, D. Schneidman-Duhovny, A. Sali, A. Nava-Tudela, and D. Fushman, "Recovering a representative conformational ensemble from underdetermined macromolecular structural data," Journal of the American Chemical Society, vol. 135, no. 44, pp. 16595-16609, 2013.
[11] D. D. Boehr, H. J. Dyson, and P. E. Wright, "An NMR perspective on enzyme dynamics," Chemical Reviews, vol. 106, no. 8, pp. 3055-3079, 2006.

[12] M. Lukin and C. de los Santos, "NMR structures of damaged DNA," Chemical Reviews, vol. 106, no. 2, pp. 607-686, 2006.

[13] S. Kang, M. M. Cetin, R. Jiang, E. S. Clevenger, and M. F. Mayer, "Synthesis of metalated pseudorotaxane polymers with full control over the average linear density of threaded macrocycles," Journal of the American Chemical Society, vol. 136, no. 36, pp. 12588-12591, 2014.

[14] X. Wang, K. Cao, Y. Liu, B. Tsang, and S. Liew, "Migration insertion polymerization (MIP) of cyclopentadienyldicarbonyldiphenylphosphinopropyliron (FpP): a new concept for main chain metal-containing polymers (MCPs)," Journal of the American Chemical Society, vol. 135, no. 9, pp. 3399-3402, 2013.

[15] V. I. Bakhmutov, "Strategies for solid-state NMR studies of materials: from diamagnetic to paramagnetic porous solids," Chemical Reviews, vol. 111, no. 2, pp. 530-562, 2011.

[16] A. Kangarlu and P.-M. L. Robitaille, "Biological effects and health implications in magnetic resonance imaging," Concepts in Magnetic Resonance, vol. 12, no. 5, pp. 321-359, 2000.

[17] S. Kunjachan, J. Ehling, G. Storm, F. Kiessling, and T. Lammers, "Noninvasive imaging of nanomedicines and nanotheranostics: principles, progress, and prospects," Chemical Reviews, vol. 115, no. 19, pp. 10907-10937, 2015.

[18] M. C. Heffern, L. M. Matosziuk, and T. J. Meade, "Lanthanide probes for bioresponsive imaging," Chemical Reviews, vol. 114, no. 8, pp. 4496-4539, 2014.

[19] R. F. Angawi, "Using a problem solving-cooperative learning approach to improve students' skills for interpreting ${ }^{1} \mathrm{H}$ NMR spectra of unknown compounds in an organic spectroscopy course," Journal of Chemical Education, vol. 91, no. 6, pp. 823829, 2014.

[20] F. Damkaci and A. Szymaniak, "Multicomponent heterocyclic chemistry for undergraduate organic laboratory: biginelli reaction with multiple unknowns," Journal of Chemical Education, vol. 91, no. 6, pp. 943-945, 2014.

[21] M. J. Fray, "Investigation of epimer formation in amidecoupling reactions: an experiment for advanced undergraduate students," Journal of Chemical Education, vol. 91, no. 1, pp. 136140, 2014.

[22] J. Hanson, B. Dasher, E. Scharrer, and T. Hoyt, "Exploring the stereochemistry of the wittig reaction: the unexpected influence of a nominal spectator ion," Journal of Chemical Education, vol. 87, no. 9, pp. 971-974, 2010.

[23] M. J. Minch, "Spin choreography: basic steps in high resolution NMR (Freeman, Ray)," Journal of Chemical Education, vol. 75, no. 2, p. 155, 1998.

[24] R. L. Ward, "A complete introduction to modern NMR spectroscopy (Macomber, Rodger S.)," Journal of Chemical Education, vol. 76, no. 4, p. 473, 1999.

[25] A. C. Dawsey, K. L. Hathaway, S. Kim, and T. J. Williams, "Introductory chemistry: a molar relaxivity experiment in the high school classroom," Journal of Chemical Education, vol. 90, no. 7, pp. 922-925, 2013.

[26] J.-P. Guégan and R. Daniellou, "An introduction to drug discovery by probing protein-substrate interactions using saturation transfer difference-nuclear magnetic interactions using Saturation Transfer Difference-Nuclear Magnetic Resonance (STD-NMR)," Journal of Chemical Education, vol. 89, no. 8, pp. 1071-1073, 2012. 
[27] E. D. Becker, High Resolution NMR Theory and Chemical Applications, Academic Press, New York, NY, USA, 2000.

[28] K. de la Vega-Hernández and M. Antuch, "The Heteronuclear Single-Quantum Correlation (HSQC) experiment: vectors versus Product Operators," Journal of Chemical Education, vol. 92, no. 3, pp. 482-487, 2015.

[29] D. P. Goldenberg, "The product operator formalism: a physical and graphical interpretation," Concepts in Magnetic Resonance Part A, vol. 36, no. 2, pp. 49-83, 2010.

[30] M. R. Bendall and T. E. Skinner, "Comparison and use of vector and quantum representations of J-coupled spin evolution in an IS spin system during RF irradiation of one spin," Journal of Magnetic Resonance, vol. 143, no. 2, pp. 329-351, 2000.

[31] J. Shriver, "Product operators and coherence transfer in multiple-pulse NMR experiments," Concepts in Magnetic Resonance, vol. 4, no. 1, pp. 1-33, 1992.

[32] D. G. Donne and D. G. Gorenstein, "A pictorial representation of product operator formalism: Nonclassical vector diagrams for multidimensional NMR," Concepts in Magnetic Resonance, vol. 9, no. 2, pp. 95-110, 1997.

[33] R. Freeman, "A physical picture of multiple-quantum coherence," Concepts in Magnetic Resonance, vol. 10, no. 2, pp. 63-84, 1998.

[34] M. H. Levitt, Spin Dynamics. Basics of Nuclear Magnetic Resonance, John Wiley \& Sons, West Sussex, UK, 2008.

[35] L. G. Hanson, "Is quantum mechanics necessary for understanding magnetic resonance?" Concepts in Magnetic Resonance Part A, vol. 32, no. 5, pp. 329-340, 2008.

[36] P. K. Mandal and A. Majumdar, "A comprehensive discussion of HSQC and HMQC pulse sequences," Concepts in Magnetic Resonance Part A, vol. 20, no. 1, pp. 1-23, 2004.

[37] J. Keeler, Understanding NMR Spectroscopy, John Wiley \& Sons, Oxford, UK, 2002.

[38] A. D. Bain, "Operator formalisms: an overview," Concepts in Magnetic Resonance Part A, vol. 28, no. 6, pp. 369-383, 2006.

[39] O. W. Sørensen, G. W. Eich, M. H. Levitt, G. Bodenhausen, and R. R. Ernst, "Product operator formalism for the description of NMR pulse experiments," Progress in Nuclear Magnetic Resonance Spectroscopy, vol. 16, pp. 163-192, 1984.

[40] N. E. Jacobsen, NMR Spectroscopy Explained. Simplified Theory, Applications and Examples for Organic Chemistry and Structural Biology, John Wiley \& Sons, Upper Saddle River, NJ, USA, 2007. 

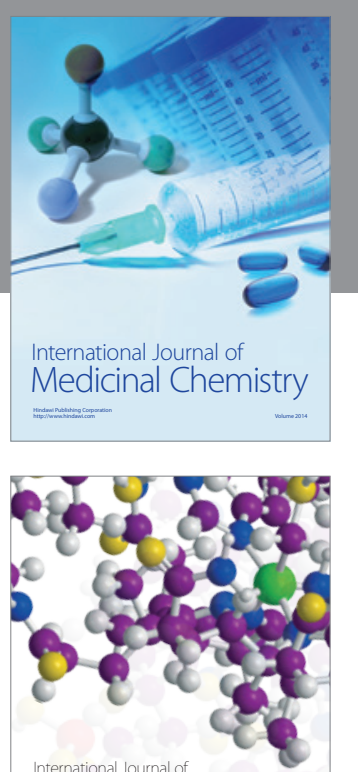

Carbohydrate Chemistry

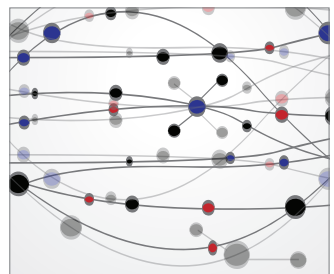

The Scientific World Journal
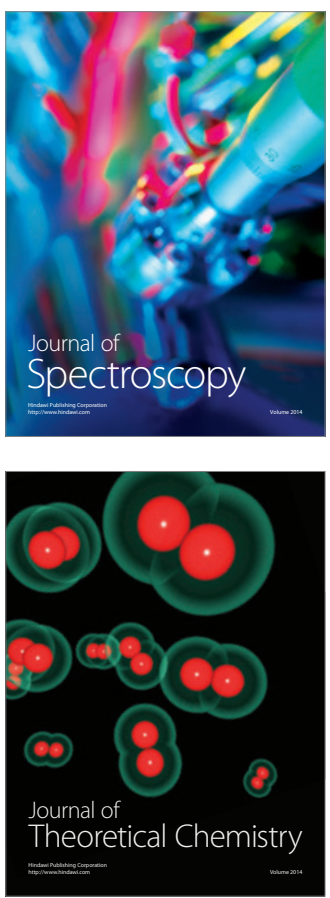
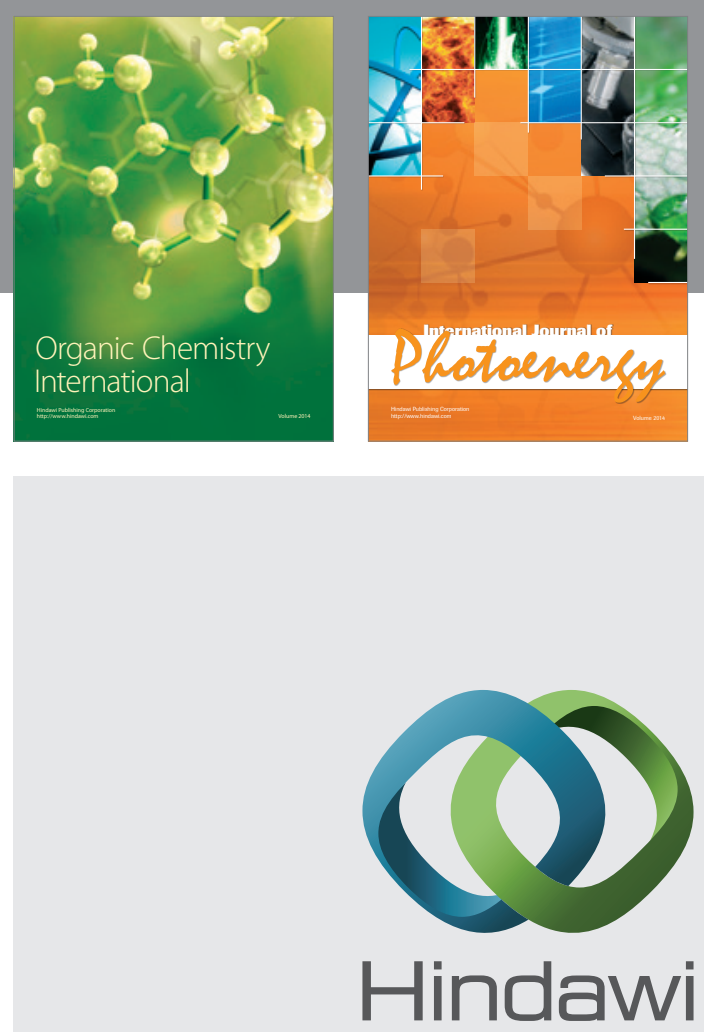

Submit your manuscripts at

http://www.hindawi.com

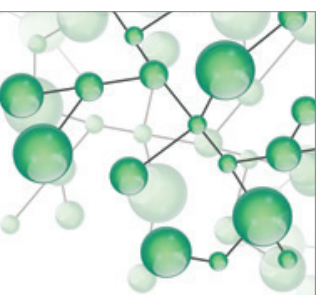

International Journal of

Inorganic Chemistry

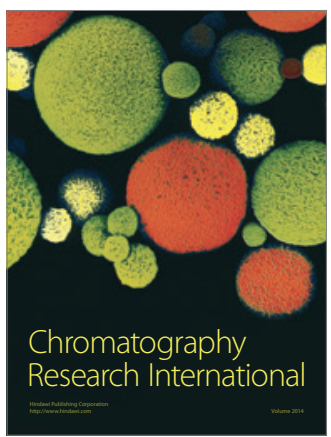

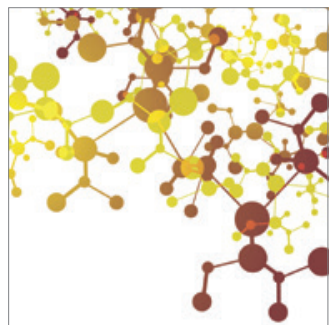

Applied Chemistry
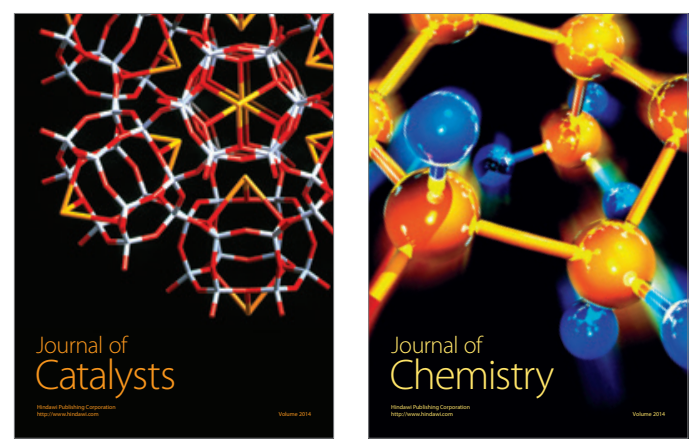
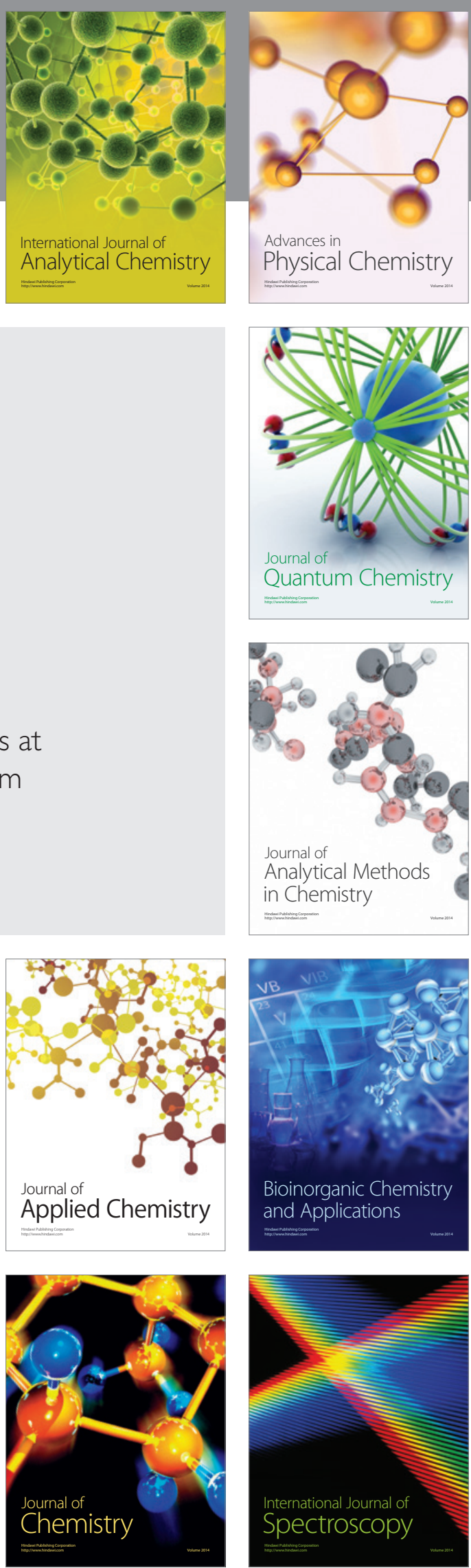